\title{
Repression of mitochondrial metabolism for cytosolic pyruvate-derived chemical production in Saccharomyces cerevisiae
}

\author{
Keisuke Morita' ${ }^{1}$ Fumio Matsuda' ${ }^{1}$ Koji Okamoto², Jun Ishii ${ }^{3,4}$, Akihiko Kondo ${ }^{3,4,5,6}$ and Hiroshi Shimizu ${ }^{*}$
}

\begin{abstract}
Background: Saccharomyces cerevisiae is a suitable host for the industrial production of pyruvate-derived chemicals such as ethanol and 2,3-butanediol (23BD). For the improvement of the productivity of these chemicals, it is essential to suppress the unnecessary pyruvate consumption in S. cerevisiae to redirect the metabolic flux toward the target chemical production. In this study, mitochondrial pyruvate transporter gene (MPC1) or the essential gene for mitophagy (ATG32) was knocked-out to repress the mitochondrial metabolism and improve the production of pyruvate-derived chemical in S. cerevisiae.

Results: The growth rates of both aforementioned strains were 1.6-fold higher than that of the control strain. ${ }^{13} \mathrm{C}$-metabolic flux analysis revealed that both strains presented similar flux distributions and successfully decreased the tricarboxylic acid cycle fluxes by 50\% compared to the control strain. Nevertheless, the intracellular metabolite pool sizes were completely different, suggesting distinct metabolic effects of gene knockouts in both strains. This difference was also observed in the test-tube culture for 23BD production. Knockout of ATG32 revealed a 23.6-fold increase in $23 B D$ titer $(557.0 \pm 20.6 \mathrm{mg} / \mathrm{L})$ compared to the control strain $(23.5 \pm 12.8 \mathrm{mg} / \mathrm{L})$, whereas the knockout of MPC1 revealed only 14.3-fold increase ( $336.4 \pm 113.5 \mathrm{mg} / \mathrm{L})$. Further investigation using the anaerobic high-density fermentation test revealed that the MPC1 knockout was more effective for ethanol production than the 23BD production.
\end{abstract}

Conclusion: These results suggest that the engineering of the mitochondrial transporters and membrane dynamics were effective in controlling the mitochondrial metabolism to improve the productivities of chemicals in yeast cytosol.

Keywords: Saccharomyces cerevisiae, Mitophagy, Metabolome, Mitochondrial pyruvate carrier, ${ }^{13} \mathrm{C}$-metabolic flux analysis, 2,3-Butanediol

\section{Background}

Yeast Saccharomyces cerevisiae is one of the suitable microbial industrial hosts for bioethanol production due to its robustness to $\mathrm{pH}$ stress and fermentation ability [1]. Moreover, it has been applied in industrial productions of various chemicals from glucose. Pyruvate produced via glycolysis acts as a key precursor for the biosynthesis of various chemicals including ethanol, lactate, and

\footnotetext{
${ }^{*}$ Correspondence: shimizu@ist.osaka-u.ac.jp

${ }^{1}$ Graduate School of Information Science and Technology, Osaka

University, 1-5 Yamadaoka, Suita, Osaka 565-0871, Japan

Full list of author information is available at the end of the article
}

2,3-butanediol (23BD). 23BD is known to be an antifreeze agent and a raw material for synthetic rubber [2-5]. In order to improve the productivity of these chemicals, it is essential to suppress the unnecessary pyruvate consumption in the S. cerevisiae cells to redirect the metabolic flux toward the target chemical production pathways.

In S. cerevisiae cells, a large amount of pyruvate is consumed by the synthesis of cell components, ethanol production, and respiration in the mitochondrion. Several studies reported that the knockout or control of ethanol biosynthesis pathway genes in S. cerevisiae improves the production of the pyruvate-derived chemicals [3, 
4, 6, 7]. In Escherichia coli, decreasing the citrate synthase expression by CRISPER/Cas9 system increased the $n$-butanol yield [8]. Moreover, a previous study reported that the repression of tricarboxylic acid (TCA) cycle by knockout of LPD1 coding the component of the pyruvate dehydrogenase and 2-oxoglutarate dehydrogenase enhanced the cytosolic isobutanol production [9]; however, few reports have emphasized on the repression of mitochondrial metabolism in yeast. In mitochondrion, the carbon loss occurs in the TCA cycle associated with the respiratory chain under aerobic conditions. In recent years, it has been reported that mitochondrial metabolic pathways function even in anaerobic fermentation conditions such as brewing for organic acid production [10]. Therefore, the repression of mitochondrial metabolism may reduce carbon loss and is presumed to improve the productivity of pyruvate-derived target chemicals.

In this study, two approaches were investigated in order to repress the mitochondrial metabolism. The first approach includes a knockout of the mitochondrial pyruvate carrier (MPC) genes responsible for pyruvate transport to mitochondria from the cytosol. Yeast has three pyruvate transporter proteins (Mpc1p, Mpc2p, and Mpc3p) that form two types of complexes for different functions. MPC complex with Mpc1p and Mpc2p acts as an active pyruvate transporter in the aerobic condition, whereas the complex with Mpc1p and Mpc3p acts as a low active transporter under anaerobic conditions [11]. Park et al. [12] reported that enhancing the MPC gene expression increased the pyruvate uptake into mitochondria and improved the isobutanol titer produced by the valine biosynthesis pathway and heterologous Ehrlich pathway in mitochondria. In this study, the knockout of $M P C 1$ was emphasized to shut-off the entrance of mitochondrial metabolism because it was essential for the formation of either complex.

The second approach includes the inhibition of mitochondrial selective degradation, termed as "mitophagy". Mitophagy is one of the autophagy mechanisms that degrade the damaged mitochondria by oxidative stress of reactive oxygen species (ROS). Molecular mechanisms of mitophagy have been actively studied using yeast as a model organism and have revealed that the Atg32p phosphorylation is indispensable for induction of mitophagy in $S$. cerevisiae [13, 14]. It has been suggested that membrane kinetics of mitophagy is also associated with fermentative metabolism. Shiroma et al. [15] reported that the ethanol production titer of ATG32 knockout strain (mitophagy inhibited yeast strain) was enhanced in the ginjo-sake brewing conditions with low nutrient. They considered that the ethanol production was increased by excess carbons from the suppression of cell growth due to the lack of reused-nutrients supplied by the mitochondrial degradation. It is presumed that metabolic effect of ATG32 knockout to increase the ethanol production can also be applied to improve other pyruvatederived chemical productions.

In this study, to enhance the productivity of pyruvate-derived chemicals in yeast by repressing the mitochondrial metabolism, knockout of MPC1 and ATG32 was carried out for the inhibition of pyruvate uptake into mitochondrion and mitophagy, respectively. The ${ }^{13} \mathrm{C}$-metabolic flux analysis $\left({ }^{13} \mathrm{C}\right.$-MFA $)$ and metabolome analysis of central carbon metabolism were performed to investigate their effect on yeast metabolism. Furthermore, in order to verify that the repression of mitochondrial metabolism was effective in improving the pyruvate-derived chemical productivity, 23BD production strains were constructed (Fig. 1). This chemical is generally preferred for yeast metabolic engineering $[4,6$, $16,17]$ and was used in this study due to its easy handling in metabolic engineering and low cell toxicity $[18,19]$.

\section{Result}

\section{Fermentation profiles of $m p c 1 \Delta$ and $\operatorname{atg} 32 \Delta$ strains} in the exponential growth phase

To investigate the effect of MPC1 and ATG32 knockout on yeast, three strains (SCM001, SCM053, and SCM003, Table 1) were obtained by introducing a plasmid pGK426 (an empty vector with $U R A 3$ marker) into the S. cerevisiae BY4742, MPC1 and ATG32 knockout strains, respectively. Strains were inoculated into $5 \mathrm{~mL}$ of SD medium containing $20 \mathrm{~g} / \mathrm{L}$ of glucose in the test tube with an initial $\mathrm{OD}_{600}=0.5$ in order to obtain the fermentation profiles in a mid-log growth phase.

Table 2 shows the growth rate, glucose consumption rate, and production rate of ethanol and glycerol in the mid-log growth phase. These values were obtained

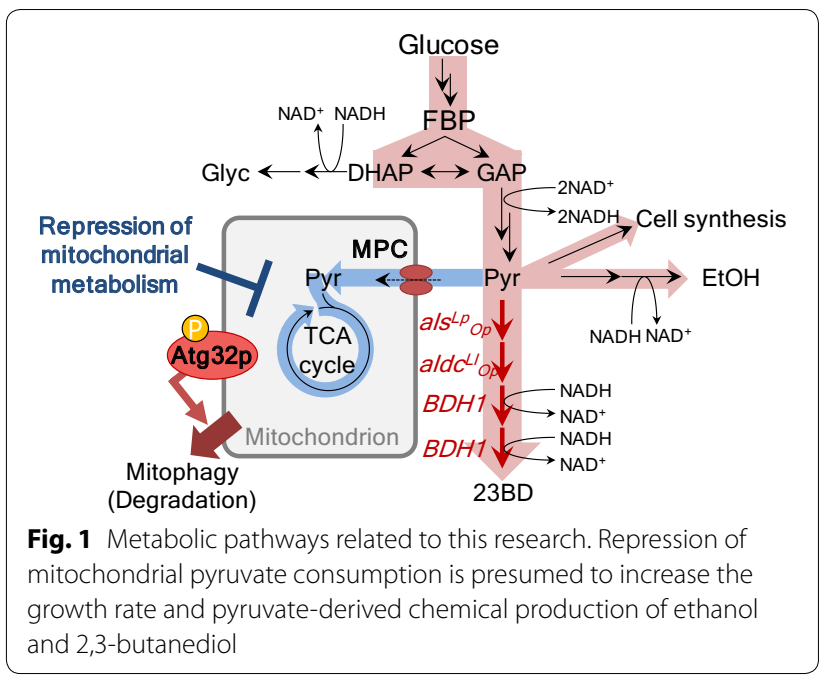


Table 1 Yeast strains and plasmids used in this study

\begin{tabular}{|c|c|c|}
\hline Strain name & Description & Source or references \\
\hline BY4742 & MATa, his $3 \Delta 1$, leu2 $\triangle 0$, lys $2 \Delta 0$, ura $3 \Delta 0$ & Thermo Scientific \\
\hline $\operatorname{mpc} 1 \Delta$ & BY4742 mpci $\triangle:: k a n M X 4$ & Thermo Scientific \\
\hline $\operatorname{atg} 32 \triangle$ & BY4742 atg32 $\because::$ kanMX4 & Thermo Scientific \\
\hline SCM001 & BY4742/pGK426 & This study \\
\hline SCM053 & $\mathrm{mpc} 1 \triangle / \mathrm{pGK} 426$ & This study \\
\hline SCM003 & $\operatorname{atg} 32 \triangle / p G K 426$ & This study \\
\hline SCM043 & BY4742/pATP426-als ${ }^{L p}{ }_{O p}$-aldc ${ }^{L I}{ }_{O p}-B D H 1$ & This study \\
\hline SCM051 & $\operatorname{mpc} 1 \triangle /$ pATP426-als ${ }^{\mathrm{Lp}}{ }_{\mathrm{Op}}$-aldc ${ }^{\mathrm{LI}}{ }_{\mathrm{Op}}-\mathrm{BDH} 1$ & This study \\
\hline SCM042 & $\operatorname{atg} 32 \triangle /$ pATP426-als ${ }^{L \mathrm{Lp}}$-aldc ${ }^{\mathrm{Ll}}{ }_{\mathrm{Op}}-\mathrm{BDH} 1$ & This study \\
\hline Plasmid & Description & Source or reference \\
\hline pGK426 & Yeast expression vector containing PGK1 promoter, $2 \mu$ origin, URA3 marker, no expression (control plasmid) & Ishii et al. [33] \\
\hline pATP426 & $\begin{array}{l}\text { Yeast three gene expression vector containing } A D H 1, T D H 3 \text {, and } P G K 1 \text { promoters, } 2 \mu \text { origin, URA3 marker, no } \\
\text { expression. }\end{array}$ & Ishii et al. [34] \\
\hline 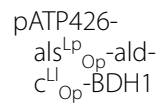 & $\begin{array}{l}\text { pATP426, co-expression of codon-optimized L. plantarum ALS, L. lactis ALDC and S. cerevisiae BDH1 genes } \\
\text { controlled by ADH1, TDH3, and PGK1 promoters }\end{array}$ & Ishii et al. [6] \\
\hline
\end{tabular}

Table 2 Growth, glucose consumption, ethanol, and glycerol production rate in BY4742 wild-type, atg32 strains

\begin{tabular}{|c|c|c|c|}
\hline & SCM001 (Wild-type) & SCM053 (mpc1 $1 \Delta)$ & SCM003 (atg32 $\Delta)$ \\
\hline Growth rate $\left(h^{-1}\right)$ & $0.15 \pm 0.01$ & $0.25 \pm 0.01$ & $0.24 \pm 0.01$ \\
\hline Glucose consumption rate $\left(\mathrm{mmol} \mathrm{gDCW}^{-1} \mathrm{~h}^{-1}\right)$ & $15.7 \pm 2.4$ & $14.8 \pm 1.1$ & $15.7 \pm 0.9$ \\
\hline Ethanol production rate $\left(\mathrm{mmol} \mathrm{gDCW}^{-1} \mathrm{~h}^{-1}\right)$ & $18.2 \pm 1.2$ & $19.9 \pm 2.0$ & $18.8 \pm 1.4$ \\
\hline Glycerol production rate $\left(\mathrm{mmol} \mathrm{gDCW}^{-1} \mathrm{~h}^{-1}\right)$ & $0.5 \pm 0.3$ & $1.3 \pm 0.1$ & $1.2 \pm 0.3$ \\
\hline
\end{tabular}

from the fermentation profile (Additional file 1: Figure S1). The growth rates of both the $m p c 1 \Delta$ and $\operatorname{atg} 32 \Delta$ strains were significantly improved $\left(0.25 \pm 0.01 \mathrm{~h}^{-1}\right.$ and $0.24 \pm 0.01 \mathrm{~h}^{-1}$, respectively) compared to that of the wild-type strain $\left(0.15 \pm 0.01 \mathrm{~h}^{-1}\right)$. Although no statistically significant difference was observed between the ethanol production rate, $m p c 1 \Delta$ and $\operatorname{atg} 32 \Delta$ strains exhibited larger value $\left(19.9 \pm 2.0 \mathrm{mmol} \mathrm{gDCW}^{-1} \mathrm{~h}^{-1}\right.$ and $18.8 \pm 1.4 \mathrm{mmol}_{\mathrm{gDCW}}{ }^{-1} \mathrm{~h}^{-1}$, respectively) than the wild-type strain $\left(18.2 \pm 1.2 \mathrm{mmol} \mathrm{gDCW}^{-1} \mathrm{~h}^{-1}\right)$. In addition, the glycerol production rates of both gene knockout strains were doubled for the wild type strains. These results clearly indicate that the knockout of MPC1 and ATG32 altered the yeast metabolism; however, similar glucose consumption rates were observed for the wild type, $m p c 1 \Delta$, and atg32 $\Delta$ strains $\left(15.7 \pm 2.4 \mathrm{mmol} \mathrm{gDCW}^{-1} \mathrm{~h}^{-1}, 14.8 \pm 1.1 \mathrm{mmol}\right.$ $\mathrm{gDCW}^{-1} \mathrm{~h}^{-1}$, and $15.7 \pm 0.9 \mathrm{mmol} \mathrm{gDCW}^{-1} \mathrm{~h}^{-1}$, respectively), suggesting that these metabolic perturbations did not occur due to the change in glucose uptake rate. These results indicated that the growth rate improvement in the $M P C 1$ or $A T G 32$ knockout strain was due to the change of the carbon flux distribution in the cell.

\section{${ }^{13} \mathrm{C}$-metabolic flux analysis of $\operatorname{atg} 32 \Delta$ and $m p c 1 \Delta$ strains}

${ }^{13} \mathrm{C}$-metabolic flux analysis $\left({ }^{13} \mathrm{C}\right.$-MFA) was carried out to investigate the distributions of the intracellular carbon fluxes. S. cerevisiae cells were cultured using $5 \mathrm{~mL}$ of SD medium containing $20 \mathrm{~g} / \mathrm{L}$ of $\left[1{ }^{13} \mathrm{C}\right]$ glucose as a sole carbon source in a test tube. Yeast cells at the mid$\log$ growth phase were harvested to obtain the hydrolyzed amino acids from the proteins. The ${ }^{13} \mathrm{C}$ enrichment of amino acids was measured via gas chromatographymass spectrometry. Metabolic flux distributions were estimated from the ${ }^{13} \mathrm{C}$ enrichment and specific rate data (Table 2) using the yeast central metabolic model presented in Additional file 1: Table S1 (see "Methods" for detailed procedure).

Figure 2 depicts the metabolic flux distribution of wild-type, $m p c 1 \Delta$, and $\operatorname{atg} 32 \Delta$ strains in the mid-log growth phase calculated by ${ }^{13} \mathrm{C}-\mathrm{MFA}$. The flux distributions were normalized to the glucose uptake rate (100\%). Most of the glucose was metabolized by the 

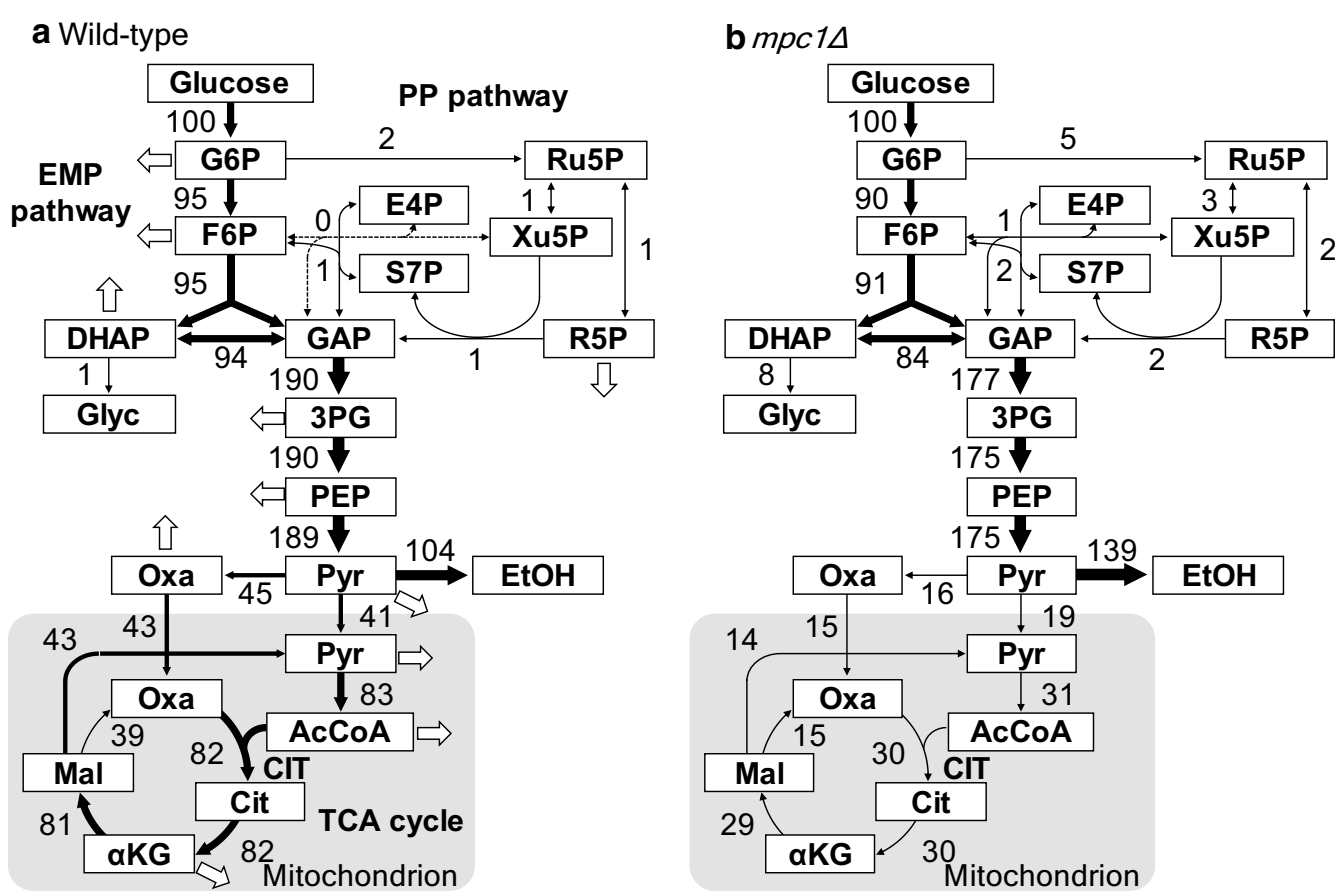

C $\operatorname{atg} 32 \Delta$

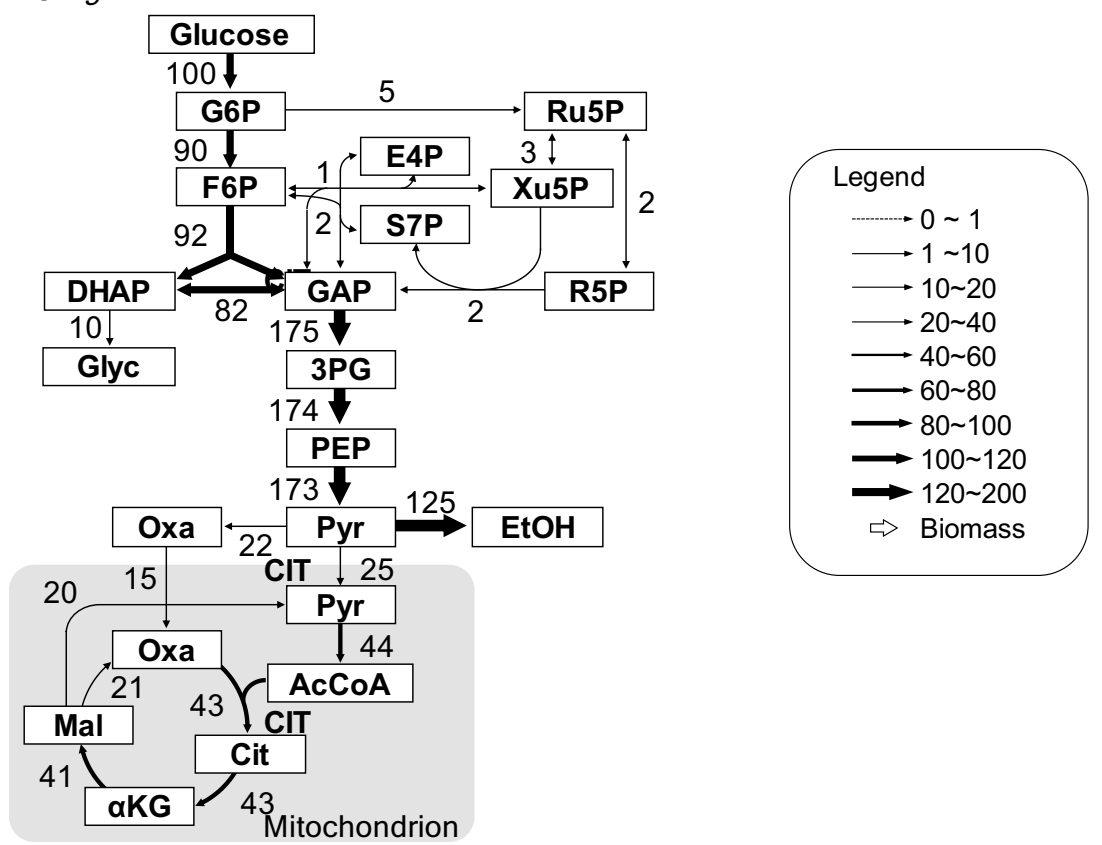

Fig. 2 Flux distributions in BY4742 wild-type, atg32 , and mpc1 $\triangle$ strains. Flux distributions are depicted for a strain SCM001 (wild-type strain), b strain SCM053 (mpc1 $\triangle$ strain) and c SCM003 strain (atg32 $\Delta$ strain) in the exponential growth phase. The flux values of the best fit were normalized to a specific glucose uptake rate of 100 . The line width reflects the flux rate

Embden-Meyerhof-Parnas pathway (EMP pathway), and the pentose-phosphate pathway (PP pathway) flux in both $m p c 1 \Delta$ strain and $\operatorname{atg} 32 \Delta$ strain increased from 2 to $5 \%$. Maximum flux changes were observed in the
TCA cycle. The metabolic flux from the acetyl-CoA in the TCA cycle was $82 \%$ in the wild-type strain; in contrast, it was reduced to $30 \%$ and $43 \%$ in the $m p c 1 \Delta$ and $\operatorname{atg} 32 \Delta$ strains, respectively. To estimate the metabolic 
flux level of the TCA cycle, the confidence interval of the citrate synthase (CIT) flux was evaluated by the grid search method. The possible ranges of the CIT flux of the wild-type, $m p c 1 \Delta$, and $\operatorname{atg} 32 \Delta$ strains were $81-83 \%$, $29-33 \%$, and $41-45 \%$, respectively. From these results, the TCA cycle flux of the $m p c 1 \Delta$ and $\operatorname{atg} 32 \Delta$ strains was clearly decreased, indicating that these gene knockouts reduced the mitochondrial metabolism and redirected the saved carbon flux to the ethanol production or biomass formations.

\section{Intracellular metabolite analysis of atg $32 \Delta$ and $m p c 1 \Delta$ strains}

In order to investigate the effect of the MPC1 and ATG32 knockout on the repression of the yeast mitochondrial metabolisms, the metabolic profiles of glycolytic intermediates after fermentation were analyzed. Cells in the mid-log growth phase were inoculated to the fresh medium with initial $\mathrm{OD}_{600}=1$ and harvested after $24 \mathrm{~h}$ for the LC-MS/MS analysis of extracted glycolytic intracellular metabolite pools.

Figure 3 presents the relative quantitation of the pool size of glycolytic metabolites for the wild-type (SCM001), $m p c 1 \Delta$, and $\operatorname{atg} 32 \Delta$ strains (SCM053 and SCM003). On comparing both knockout strains, with similar changes in the metabolic flux distributions, the knockout of MPC1 and ATG32 caused distinct effects on the intracellular pool size. Knockout of MPC1 indicated a 2.1fold increase in fructose 1,6-bisphosphate (FBP) and $50 \%$ decrease in phosphoenol pyruvate (PEP) compared to the wild-type strain. Since FBP is an allosteric activator of pyruvate kinase (PK) that converts PEP to pyruvate [20], the increased FBP concentration led to high PK activity, resulting in reduced PEP concentration. This was in accordance with the growth increase and ethanol production. Moreover, the acetyl-CoA pool size was reduced in $m p c 1 \Delta$ strain suggesting that the knockout of $M P C 1$ inhibited the mitochondrial pyruvate uptake and metabolism.

In contrast, intracellular acetyl-CoA pool size was also decreased, the pyruvate accumulation was observed in $\operatorname{atg} 32 \Delta$ strain (2.3-fold higher than that of the control strain). Moreover, the NADPH pool size decreased by $0.4-$ fold. Because the NADPH was consumed as a response to oxidative stress, the degree of ROS activities was measured using a fluorescent reagent (Additional file 1: Figure S2). No change was observed in the fluorescence intensity of the control and $m p c 1 \Delta$ strains (733 \pm 35 and $752 \pm 36$, respectively). Nevertheless, as the fluorescence intensity increased in atg32 $2 \Delta$ strain $(929 \pm 36)$, the increase in ROS activity was confirmed. Therefore, in the atg32 $\Delta$ strain, NADPH was consumed by response to oxidative stress, despite the increase of high PP pathway flux. On the other hand, no oxidative stress was observed in the mpc1 $1 \Delta$ strain, in which the NADPH pool was increased. Thus, although both mutant strains displayed similar flux distribution in their central metabolism, the differences in the responses to oxidative stress and fluxes that were not included in the central metabolism were likely to affect the metabolite pool sizes. Furthermore, in the atg32 $\Delta$ strain, mitochondrial metabolic activities decreased because of the oxidative stress due to the inhibition of mitophagy for the degradation of malfunctioning mitochondria. These findings were consistent with the decrease in TCA cycle flux and pyruvate accumulation.

\section{Application of intracellular excess pyruvate to 2,3-butanediol production}

Both gene knockout of $M P C 1$ and ATG32 were used for repressing the mitochondrial metabolism. To investigate the effect of these gene knockouts on the cytosolic production of pyruvate-derived chemicals, 23BD producing yeast strains were constructed. The 23BD is biologically synthesized from two molecules of pyruvate by four-step reactions catalyzed by acetolactate synthase (ALS), acetolactate decarboxylase (ALDC), and butanediol dehydrogenase (BDH). In this study, a plasmid constructed in the previous study (pATP426-als ${ }^{\mathrm{Lp}}{ }_{\mathrm{Op}}$-aldc ${ }_{\mathrm{Op}}^{\mathrm{Ll}}-\mathrm{BDH} 1$, als ${ }_{O p}^{L p}$, $a c d c^{L l}{ }_{O p}, B D H 1$ : codon-optimized ALS and ALDC genes derived from Lactobacillus plantarum and Lactococcus lactis and the BDH genes derived from S. cerevisiae [6]) was introduced in S. cerevisiae BY4742 (SCM043 strain) for cytosolic 23BD production.

The strains were cultivated in $5 \mathrm{~mL}$ of SD medium in test tubes with initial $\mathrm{OD}_{600}$ value as 1 (Fig. 4). The 23BD titer of SCM043 strain harboring a plasmid pATP426$\mathrm{als}^{\mathrm{Lp}}{ }_{\mathrm{Op}}-\mathrm{aldc}^{\mathrm{Ll}}{ }_{\mathrm{Op}}-\mathrm{BDH} 1$ was $23.5 \pm 12.8 \mathrm{mg} / \mathrm{L}$. The additional knockout of MPC1 and ATG32 (SCM051 and SCM042) increased the 23BD production strain titer by 14.3 -fold and 23.6 -fold $(336.4 \pm 113.5 \mathrm{mg} / \mathrm{L}$ and $557.0 \pm 20.6 \mathrm{mg} / \mathrm{L}$, respectively). In contrast, the final $\mathrm{OD}_{600}$ values decreased to $3.3 \pm 0.3$ and $2.6 \pm 0.4$ when compared to the SCM043 strain (4.2 \pm 0.1$)$. These results indicated that the ATG32 knockout was more effective than the MPC1 knockout for 23BD production, although both gene knockouts could redirect the saved carbon flux of mitochondria during $23 \mathrm{BD}$ production.

\section{Growth-arrested high-density fermentation for 23BD production}

Anaerobic high-density fermentation was carried out in $50 \mathrm{~mL}$ culture of $100 \mathrm{~g} / \mathrm{L}$ glucose SD medium with an initial $\mathrm{OD}_{600}=20$ (Fig. 5). Cell growth was arrested in this condition since the $\mathrm{OD}_{600}$ value was not significantly changed during the $72 \mathrm{~h}$ of fermentation. The 


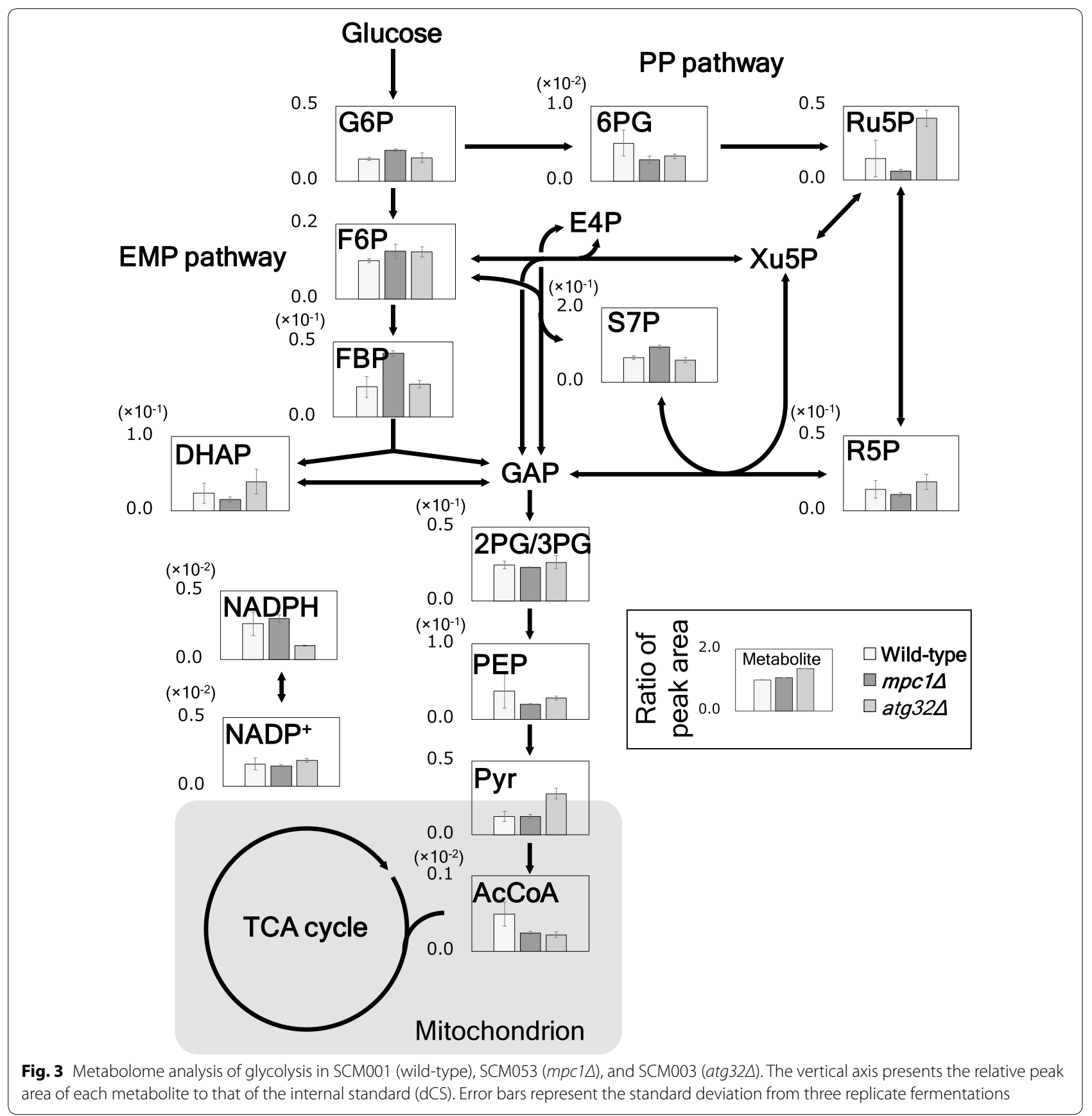

entire glucose was consumed at $48 \mathrm{~h}$; the 23BD titer of the control strain (SCM043 strain) at $72 \mathrm{~h}$ culture was $3352.6 \pm 263.9 \mathrm{mg} / \mathrm{L}$. SCM042 strain improved 23BD titer to $5452.6 \pm 359.8 \mathrm{mg} / \mathrm{L}$ by $A T G 32$ knockout; however, knockout of MPC1 decreased the titer to $2184.4 \pm 796.8 \mathrm{mg} / \mathrm{L}$ (SCM051 strain). In contrast, $m p c 1 \Delta$ strain indicated $42.2 \pm 2.8 \mathrm{~g} / \mathrm{L}$ of ethanol production titer compared to the control strain $(37.4 \pm 4.4 \mathrm{~g} / \mathrm{L})$ and the $\operatorname{atg} 32 \Delta$ strain $(33.1 \pm 1.3 \mathrm{~g} / \mathrm{L})$.

\section{Discussion}

Herzig et al. and Bricker et al. [21, 22] reported that the growth of $m p c 1 \Delta$ strain was improved by the plate cultures with supplementation of leucine and valine. However, in this study, the $m p c 1 \Delta$ and atg $32 \Delta$ strains grew better compared to the wild type strain in test tube culture when supplemented only with leucine. Knockout of MPC1 was confirmed by PCR (Additional file 1: Figure S3). Therefore, we conducted a drop test to compare the discrepancy of growth abilities between our mutants and 


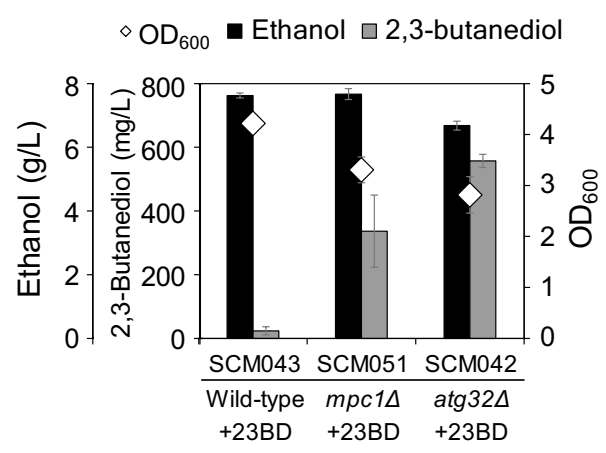

Fig. 4 Test-tube scale culture of SCM043 (wild-type), SCM051 (mpc1 $\triangle$ ), SCM042 (atg32 $\triangle$ ) strains for 2,3-butanediol fermentation. The black and gray bars represent ethanol and 2,3-butanediol titers, respectively. $+23 \mathrm{BD}$ denotes the strains harboring the plasmid pATP426-als ${ }^{\mathrm{Lp}} \mathrm{Op}^{-}$-aldd ${ }^{\mathrm{L}}{ }_{\mathrm{Op}}-\mathrm{BDH} 1$ for $23 \mathrm{BD}$ production. Open diamonds represent the $\mathrm{OD}_{600}$ of strains. Each value represents the standard deviation of three replicate fermentations

mutants of prior studies (Additional file 1: Figure S4). The growth of the $M P C 1$ mutant was improved by the addition of $100 \mathrm{mg} / \mathrm{L}$ valine and $100 \mathrm{mg} / \mathrm{L}$ leucine compared to the wild type strain. The MPC1 mutant supplemented with valine and leucine behaved similarly as previously described. However, the growth rate of the control strains was markedly different, probably due to the difference of strains and unexpected mutations. Therefore, we conclude that the growth of our $m p c 1 \Delta$ strain was not different from previous studies' growth.

In the present study, ${ }^{13} \mathrm{C}$-MFA was carried out to investigate the metabolic perturbations in $m p c 1 \Delta$ and atg32 $\Delta$ strains. The carbon fluxes of the TCA cycle were successfully suppressed (Fig. 2) and redirected for cell growth (Table 2). Although the reduction in the TCA cycle flux and the increased growth rate contradicted each other, yeast metabolisms were considered as anaerobic reactions due to the Crabtree effect as the strains were cultured in $20 \mathrm{~g} / \mathrm{L}$ of glucose SD medium. Thus, in the control strain, TCA cycle presumably worked with alternative respiration, which produced water by transferring electrons from NADH directly to oxygen without the electron transport chain and ATP generation. The alternative respiration also decreased the ethanol fermentation ability [23]. In these strains, the glycolysis acted as an ATP producer for growth improvement, and the decrease in TCA cycle flux increased the carbon quantity necessary for biomass formation.

The knockout of MPC1 increased cytosolic 23BD production in test-tube scale fermentation coupled with cell growth (Fig. 4). Enhanced MPC1 and MPC2 expression increased the mitochondrial isobutanol titer in the previous study [12]. In the present study, MPC knockout indicated that it was effective in improving the pyruvate-derived chemical production in the cytosolic biosynthesis pathway. This result suggested that both the expression and knockout of MPC genes are essential for
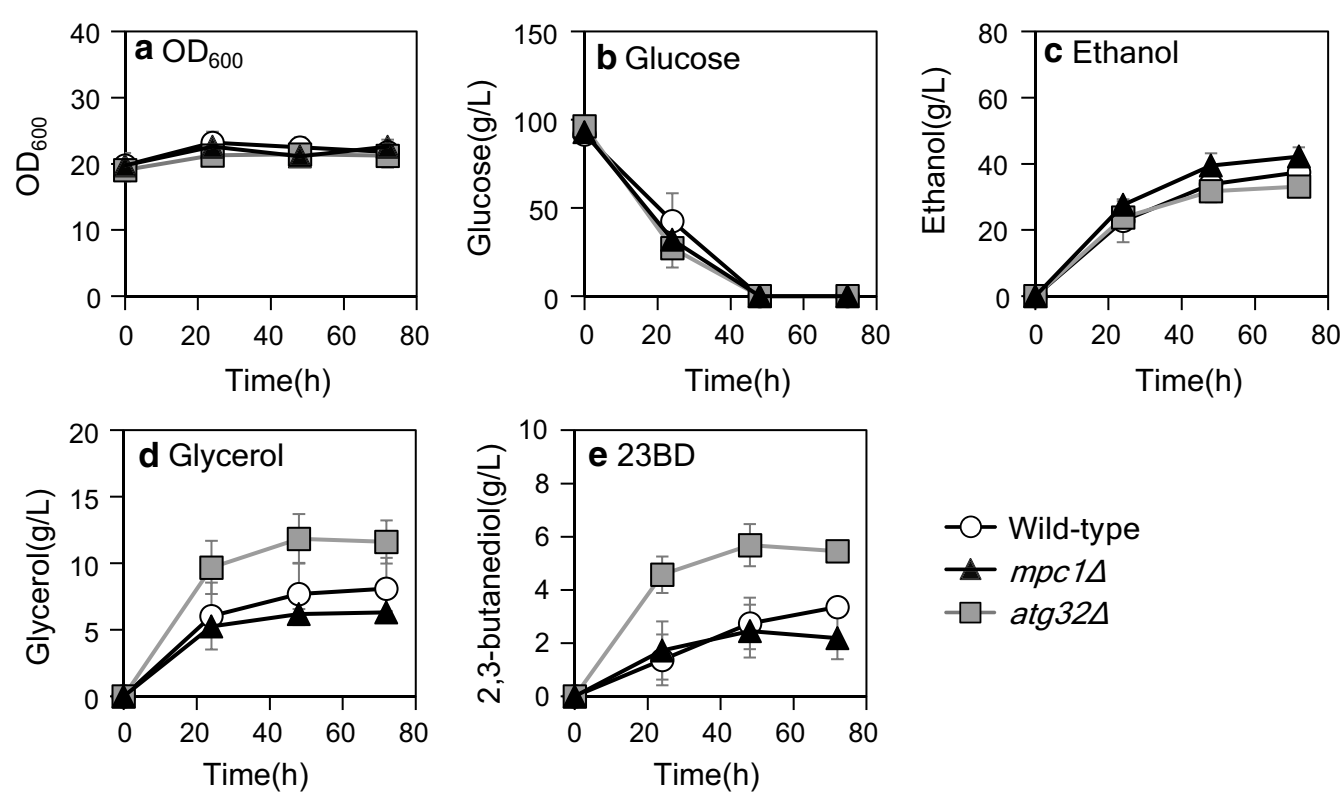

Fig. 5 High-density fermentation test of 2,3-butanediol production strains. a $\mathrm{OD}_{600}$ and $\mathbf{b}$ glucose, c ethanol, d glycerol, and e 2,3-butanediol

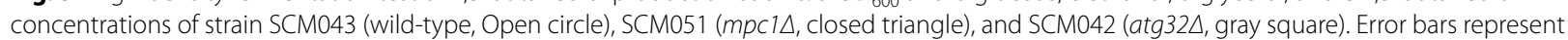
the standard deviations from three replicate fermentations 
yeast metabolic engineering in certain situations; however, the ethanol titer was increased in high-density fermentation compared to the 23BD titer (Fig. 5e). These results suggested that there were competitions of the pyruvate utilization for biomass synthesis, ethanol, and $23 \mathrm{BD}$ production. In the $m p c 1 \Delta$ strain, the Crabtree effect may have strongly enhanced and activated ethanol production under the high-density anaerobic condition. Therefore, the expression of powerful enzymes for 23BD production or decreased activity of the ethanol biosynthesis pathway was required for the improvement of 23BD titer in anaerobic conditions.

Shiroma et al. [15] reported that improvement in the ethanol titer in Sake brewing ATG32 knockout strain occurred due to decreased biomass synthesis owing to lack of the reuse of nutrient sources available after the mitochondrial degradation. Nevertheless, in the present study, the relation between the growth rate and the ethanol production was not consistent, indicating the contribution of reduced mitochondrial flux for the increase of growth abilities (Table 2, Fig. 2). 23BD fermentation test also indicates that the distribution of the pyruvate consumption flux in atg32 $\Delta$ strain was controlled by the delicate balance between the pathways and influenced sensitively by tugging power of metabolic enzymes like $m p c 1 \Delta$ strain. However, the influence of the Crabtree effect in the $\operatorname{atg} 32 \Delta$ strain was reduced under anaerobic conditions of high-density culture compared to the mpc1s strain. This might be due to the preservation of the pyruvate transportability in the atg32 $2 \Delta$ strain, wherein ethanol production was suppressed by trapping of pyruvate in mitochondria, followed by the conversion of pyruvate to acetolactate by the endogenous ALS activity encoded by $I L V 2$, an intermediate of $23 \mathrm{BD}$.

\section{Conclusion}

In the present study, strategies for the repression of mitochondrial metabolism, knockout of the mitochondrial pyruvate carrier gene (MPC1), and the essential gene for mitophagy (ATG32) were assessed in order to improve the pyruvate-derived chemical productivity in yeast. Both gene knockouts successfully suppressed the carbon flux of TCA cycle present in the mitochondria and redirected the carbon flux to the cell growth. The analysis of the intracellular metabolites suggested that the knockout of $M P C 1$ inhibited mitochondrial pyruvate uptake and metabolism, whereas that of ATG32 decreased mitochondrial metabolism by oxidative stress due to the mitophagy inhibition. These findings were applied in the cytosolic 23BD production and it was confirmed that both gene knockouts were effective in improving the production titers. The results of this study suggested that the new approaches in metabolic engineering involving the mitochondrial transporters and membrane dynamics are effective in controlling the metabolism to improve the cytosolic target chemical productivity.

\section{Methods}

Strains, plasmids, and yeast transformation

The yeast strains and plasmids used in this study are

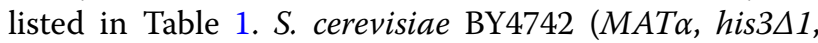
leu $2 \Delta 0$, lys $2 \Delta 0$, ura $3 \Delta 0$; purchased from Thermo Scientific, Pittsburgh, PA, USA) was used as the yeast host strain. Plasmids were derived from the pGK and pATP vectors, in which the gene expression is controlled by the $P G K 1, A D H 1$, and TDH3 promoters [21, 22].

\section{Culture conditions}

All strains were cultured in yeast extract peptone dextrose (YPD) medium (10 g/L bacto yeast extract, $20 \mathrm{~g} / \mathrm{L}$ bacto peptone, $20 \mathrm{~g} / \mathrm{L}$ glucose) and synthetic dextrose (SD) medium $(67 \mathrm{~g} / \mathrm{L}$ yeast nitrogen base without amino acids and $20 \mathrm{~g} / \mathrm{L}$ glucose, as necessary, $60 \mathrm{mg} / \mathrm{L}$ leucine, $30 \mathrm{mg} / \mathrm{L}$ lysine hydrochloride, $20 \mathrm{mg} / \mathrm{L}$ histidine, and $20 \mathrm{mg} / \mathrm{L}$ uracil). In the carbon labeling experiments, $\mathrm{SD}$ medium $(67 \mathrm{~g} / \mathrm{L}$ yeast nitrogen base without amino acids and $20 \mathrm{~g} / \mathrm{L}\left[1-{ }^{13} \mathrm{C}\right]$ glucose) containing the required amino acids was used. $\left[1-{ }^{13} \mathrm{C}\right]$ glucose $(99 \%)$ was purchased from Cambridge Isotope Laboratories (Andover, MA, USA). Yeast cells from the plate medium were cultured in $5 \mathrm{~mL}$ of SD medium containing the required amino acids at $30^{\circ} \mathrm{C}$ by shaking at $150 \mathrm{rpm}$. In the main culture, cells were incubated with similar conditions. The initial $\mathrm{OD}_{600}$ values were set at 0.5 . The $\mathrm{OD}_{600}$ values were determined via a spectrophotometer (UVmini-1240, Shimadzu, Kyoto, Japan). For the fermentation test, transformants were cultured for $72 \mathrm{~h}$ at $30{ }^{\circ} \mathrm{C}$ by shaking at $150 \mathrm{rpm}$ in $5 \mathrm{~mL}$ of SD medium containing $20 \mathrm{~g} / \mathrm{L}$ glucose and required amino acids. The initial $\mathrm{OD}_{600}$ values for the main cultures were set at 1.0.

For high-density fermentation, cells were inoculated into $50 \mathrm{~mL}$ of synthetic medium $(67 \mathrm{~g} / \mathrm{L}$ yeast nitrogen base without amino acids and $100 \mathrm{~g} / \mathrm{L}$ glucose, as necessary, $60 \mathrm{mg} / \mathrm{L}$ leucine, $30 \mathrm{mg} / \mathrm{L}$ lysine hydrochloride, $20 \mathrm{mg} / \mathrm{L}$ histidine, and $20 \mathrm{mg} / \mathrm{L}$ uracil). The initial $\mathrm{OD}_{600}$ values were set at 20. Fermentation was carried out at $30^{\circ} \mathrm{C}$ with mild agitation in $100-\mathrm{mL}$ closed bottles equipped with a bubbling $\mathrm{CO}_{2}$ outlet.

\section{Analysis of extracellular metabolites}

To determine the concentrations of glucose, ethanol, acetate, glycerol, and pyruvate in the culture medium, supernatant was obtained by centrifugation at $15,000 \mathrm{rpm}$ at $4{ }^{\circ} \mathrm{C}$ for 5 min and was subjected to gas chromatography 
(GC; Agilent 7890A GC; Agilent Technologies, Santa Clara, USA) and high-performance liquid chromatography (HPLC) system (Shimadzu, Japan). The GC was operated with the following conditions: column, Stabilwax $60 \mathrm{~m} \times 0.32 \mathrm{~mm} \mathrm{ID} \times 1 \mu \mathrm{m}$ (Restek, Bellefonte, USA); carrier gas, helium; flow rate, $6.5 \mathrm{~mL} / \mathrm{min}$; injection volume, $1 \mu \mathrm{L}$; split ratio, $1: 10$; oven temperature, $70{ }^{\circ} \mathrm{C}$ for $3 \mathrm{~min}$ and raised at $10{ }^{\circ} \mathrm{C} / \mathrm{min}$; flame ionization detector (FID) temperature, $250{ }^{\circ} \mathrm{C}$. The HPLC system was equipped with an Aminex HPX-87H column $(7.8 \mathrm{~mm}$, $300 \mathrm{~mm}$, Bio-Rad, USA), UV/vis detector (SPD-20A, Shimadzu, Kyoto, Japan), and refractive index detector (RID10A, Shimadzu, Kyoto, Japan). The column temperature was set at $65{ }^{\circ} \mathrm{C}$, and $1.5 \mathrm{mM} \mathrm{H}_{2} \mathrm{SO}_{4}$ was used as the mobile phase with a flow rate of $0.5 \mathrm{~mL} \mathrm{~min}^{-1}$. The flow cell temperature of the refractive index detector was set at $40{ }^{\circ} \mathrm{C}$.

\section{${ }^{13} \mathrm{C}$-metabolic flux analysis $\left({ }^{13} \mathrm{C}-\mathrm{MFA}\right)$}

To analyze the ${ }^{13} \mathrm{C}$ label patterns of amino acids derived from cellular proteins, the cells in the exponential growth phase were collected via centrifugation at 15,000 rpm at $4{ }^{\circ} \mathrm{C}$ for $5 \mathrm{~min}$. The cell pellet was washed twice with $0.9 \% \mathrm{NaCl}$ and was hydrolyzed in $2 \mathrm{~mL} 6 \mathrm{~N} \mathrm{HCl}$ at $105^{\circ} \mathrm{C}$ for $18 \mathrm{~h}$. After filtration (Cosmonice Filter W; pore size, $0.45 \mu \mathrm{m}$; filter diameter, $13 \mathrm{~mm}$; Nacalai Tesque, Kyoto, Japan), $10 \mu \mathrm{L}$ of the internal standard (600 $\mu \mathrm{M}$ cycloleucine) was added to the hydrolysate and evaporated to dryness. The dried residue was dissolved in $50 \mu \mathrm{L}$ acetonitrile and $50 \mu \mathrm{L} \quad N$-(tert-butyldimethylsilyl)$N$-methyl-trifluoroacetamide containing $1 \%$ tert-butyldimethylchlorosilane and incubated at $105^{\circ} \mathrm{C}$ for $1 \mathrm{~h}$. After cooling overnight, the supernatant was subjected to gas chromatography-mass spectrometry (GC-MS) analysis following a previously described method [24]. The metabolic network of $S$. cerevisiae used the previously published models [25]. The metabolic model includes the major pathways of central carbon metabolism (glycolysis, PP pathway, anaplerosis, and tricarboxylic acid cycle) and the transport reaction between cytosol and mitochondria. Pyruvate, oxaloacetate, and acetyl-CoA (AcCOA) in the cytosol and mitochondria are considered as separate metabolite pools.

The computational procedure was performed by OpenMebius software [26] using MATLAB2013a (MathWorks, Natick, MA, USA). The metabolic flux distribution was estimated by nonlinear fitting of the metabolic model using the isotopic labeling patterns of proteinogenic amino acids determined by GC-MS. The nonlinear optimization was performed by using "fmincon" function in MATLAB optimization toolbox (MathWorks, Natick, MA, USA).
A 95\% confidence interval of citrate synthase (CIT) flux was calculated by a grid search method following a previously described method [25, 27-29].

\section{Liquid chromatography-tandem-mass spectrometry (LC- MS/MS) analysis of intermediate metabolites}

Cells in the exponential growth phase were inoculated into a fresh medium with initial $\mathrm{OD}_{600}=1$ and were harvested after $24 \mathrm{~h}$ for the LC-MS/MS analysis of extracted glycolytic intracellular metabolite pools $\left(\left[\mathrm{OD}_{600} \quad\right.\right.$ value $] \times[$ volume $\left.(\mathrm{mL})]=20\right)$. The culture broth was sampled rapidly and filtered through a filter of $0.5 \mu \mathrm{m}$ pore size (PTFE-type membrane, Advantec, Tokyo, Japan). Cells on the filter were immediately immersed in $1.6 \mathrm{~mL}$ methanol with $10 \mathrm{mM}$ of 10 -camphor sulfonic acid $\left(\mathrm{dCS} ;-80{ }^{\circ} \mathrm{C}\right)$ and were preserved at $-80{ }^{\circ} \mathrm{C}$ until further extraction [30, 31]. Metabolites were extracted by adding $1.6 \mathrm{~mL}$ of chloroform $\left(-30{ }^{\circ} \mathrm{C}\right)$ and $640 \mu \mathrm{L}$ of Milli-Q water $\left(4{ }^{\circ} \mathrm{C}\right)$ and vortexed for $1 \mathrm{~min}$; thereafter, the mixture was centrifuged at $4500 \times g$ for $40 \mathrm{~min}$ at $4{ }^{\circ} \mathrm{C}$. Two hundred and fifty microliters of aqueous layer were dispensed into a 1.5$\mathrm{mL}$ tube. The extract solution was dispensed into five Eppendorf tubes and dried using a SpeedVac SPD1010 (Thermo Fisher Scientific, Waltham, MA, USA) at room temperature. The dried samples were suspended in $50 \mu \mathrm{L}$ of Milli-Q water.

LC-MS/MS analysis (LC: Agilent 1200 infinity series; Agilent Technologies, Santa Clara, CA, USA; MS/MS: API 3200; AB SCIEX, MA, USA) was performed under the following conditions [32]: column, ProteCol-P C18 HQ103 $(2.1 \times 150 \mathrm{~mm}$, particle size of $3 \mu \mathrm{m})$; mobile phase, $10 \mathrm{mM}$ tributylamine/15 $\mathrm{mM}$ acetic acid in water (A) and methanol (B); flow rate: $0.2 \mathrm{~mL} \mathrm{~min}^{-1}$; gradient curve, $100 \% \mathrm{~A}: 0 \% \mathrm{~B}$ at $0 \mathrm{~min}, 10 \% \mathrm{~A}: 90 \% \mathrm{~B}$ at $24 \mathrm{~min}, 100 \% \mathrm{~A}: 0 \% \mathrm{~B}$ at $24.1 \mathrm{~min}$, and $100 \% \mathrm{~A}: 0 \%$ $\mathrm{B}$ at $30 \mathrm{~min}$; injection volume, $3 \mu \mathrm{L}$; column temperature, $35{ }^{\circ} \mathrm{C}$; mode of mass analysis, negative ion mode; nebulizer flow, 55 psi; dry gas flow rate, $10 \mathrm{~L} \mathrm{~min}^{-1}$; sheath gas flow rate, $11 \mathrm{~L} \mathrm{~min}^{-1}$; dry gas temperature, $300{ }^{\circ} \mathrm{C}$; sheath gas temperature, $380{ }^{\circ} \mathrm{C}$; capillary voltage, $3.5 \mathrm{kV}$. The parameters for selected reaction monitoring (SRM) of target metabolites have been specified in Additional file 1: Table S2. The peak of each target metabolite was identified by comparing its chromatographic behavior with that of an authentic standard. The peak area was determined using Analyst software (version 1.6.2, AB SCIEX). Relative quantitation was performed based on the MS data using the ratio of each metabolite's peak areas to the dCS peak area. 


\section{Measurement of intracellular oxidative stress of the yeast cell}

Intracellular oxidative stress was measured using an OxiSelect $^{\mathrm{TM}}$ Intracellular ROS assay kit (Cell Biolabs Inc.). Cells were harvested $\left(\mathrm{OD}_{600} \times \mathrm{mL}=1\right)$ and centrifuged. The cell pellet was washed by $1 \mathrm{~mL}$ of PBS buffer twice and incubated in $1 \mathrm{~mL}$ of SD medium containing $40 \mu \mathrm{L}$ of 2,7-dichlorodihydrofluorescin diacetate (DCFH-DA), $30{ }^{\circ} \mathrm{C}$ for $60 \mathrm{~min}$. Cells were harvested and washed by PBS again and were then suspended in $100 \mu \mathrm{L}$ of PBS on the 96 well plate for measuring oxidized DCF green fluorescent.

\section{Supplementary information}

Supplementary information accompanies this paper at https://doi. org/10.1186/s12934-019-1226-6.

\section{Additional file 1: Additional tables and figures.}

\section{Abbreviations}

G6P: glucose 6-phosphate; F6P: fructose-6-phosphate; FBP: fructose-1,6-bisphosphate; DHAP: dihydroxyacetone phosphate; GAP: glyceraldehyde 3-phosphate; 3PG: 3-phosphoglycerate; 2PG: 2-phosphoglycerate,; PEP: phosphoenol pyruvate; Pyr: pyruvate; AcCoA: acetyl-CoA; Ru5P: ribulose-5-phosphate; Xu5P: xylose-5-phosphate; R5P: ribose 5-phosphate; E4P: erythrose-4-phosphate; S7P: sedoheptulose-7-phosphate; aKG: a-ketoglutarate; Mal: malate; Oxa: oxaloacetate; CIT: citrate synthase; EMP pathway: Embden-MeyerhofParnas pathway; PP pathway: pentose-phosphate pathway; MPC: mitochondrial pyruvate carrier; TCA cycle: tricarboxylic acid cycle; EtOH: ethanol; Glyc: glycerol; 23BD: 2,3-butanediol.

\section{Acknowledgements}

We thank Dr. Yoshihiro Toya for his support in performing ${ }^{13} \mathrm{C}-\mathrm{MFA}$. pGK series vectors were provided by the National Bio-Resource Project (NBRP), Japan.

\section{Authors' contributions}

KM designed and carried out all experiments, and drafted the manuscript. FM, $\mathrm{KO}, \mathrm{Jl}$, and AK revised the manuscript and further strengthened the interpretation of the data. HS supervised and coordinated the research, and revised the manuscript. All authors read and approved the final manuscript.

\section{Funding}

This research was supported by the Industrial Technology Research Grant Program for 2011 of the New Energy and Industrial Technology Development Organization (NEDO), and Grants-in-Aid for Scientific Research (C) (Grant No. 18K04851). This research was also supported by the "Program for Leading Graduate Schools" of the Ministry of Education, Culture, Sports, Science and Technology, Japan.

\section{Availability of data and materials}

The datasets used in the current study are available from the corresponding author on reasonable request.

\section{Ethics approval and consent to participate Not applicable.}

\section{Consent for publication}

Not applicable.

\section{Competing interests}

The authors declare that they have no competing interests.

\section{Author details}

${ }^{1}$ Graduate School of Information Science and Technology, Osaka University, 1-5 Yamadaoka, Suita, Osaka 565-0871, Japan. ${ }^{2}$ Graduate School of Frontier Bioscience, Osaka University, 1-3 Yamadaoka, Suita, Osaka 565-0871, Japan. ${ }^{3}$ Engineering Biology Research Center, Kobe University, 1-1 Rokkodai, Nada, Kobe, Hyogo 657-8501, Japan. ${ }^{4}$ Graduate School of Science, Technology and Innovation, Kobe University, 1-1 Rokkodai, Nada, Kobe, Hyogo 657-8501, Japan. ${ }^{5}$ Department of Chemical Science and Engineering, Graduate School of Engineering, Kobe University, 1-1 Rokkodai, Nada, Kobe, Hyogo 657-8501, Japan. ${ }^{6}$ RIKEN Center for Sustainable Resource Science, 1-7-22 Suehiro, Tsurumi, Yokohama, Kanagawa 230-0045, Japan.

Received: 22 March 2019 Accepted: 9 October 2019

Published online: 15 October 2019

\section{References}

1. Hong KK, Nielsen J. Metabolic engineering of Saccharomyces cerevisiae: a key cell factory platform for future biorefineries. Cell Mol Life Sci. 2012;69:2671-90.

2. Hjersted JL, Henson MA, Mahadevan R. Genome-scale analysis of Saccharomyces cerevisiae metabolism and ethanol production in fed-batch culture. Biotechnol Bioeng. 2007;97(5):1190-204.

3. Ida Y, Hirasawa T, Furusawa C, Shimizu H. Utilization of Saccharomyces cerevisiae recombinant strain incapable of both ethanol and glycerol biosynthesis for anaerobic bioproduction. Appl Microbiol Biotechnol. 2013;97:4811-9.

4. Kim SJ, Seo SO, Jin YS, Seo JH. Production of 2,3-butanediol by engineered Saccharomyces cerevisiae. Bioresour Technol. 2013;146:274-81.

5. Lynch J. BD monomer and elastomer production processes. Chem-Biol Interact. 2001;135:147-53.

6. Ishii J, Morita K, Ida K, Kato H, Kinoshita S, Hataya S, Shimizu H, Kondo A, Matsuda F. A pyruvate carbon flux tugging strategy for increasing 2,3-butanediol production and reducing ethanol subgeneration in the yeast Saccharomyces cerevisiae. Biotechnol Biofuels. 2018;11:180.

7. Zhao EM, Zhang Y, Mehl J, Park H, Lalwani MA, Toettcher JE, Avalos JL. Optogenetic regulation of engineered cellular metabolism for microbial chemical production. Nature. 2018;555(7698):683-7.

8. Heo MJ, Jung HM, Um J, Lee SW, Oh MK. Controlling citrate synthase expression by CRISPR/Cas9 genome editing for $n$-butanol production in Escherichia coli. ACS Synth Biol. 2017;6(2):182-9.

9. Matsuda F, Ishii J, Kondo T, Ida K, Tezuka H, Kondo A. Increased isobutanol production in Saccharomyces cerevisiae by eliminating competing pathways and resolving cofactor imbalance. Microb Cell Fact. 2013;12:119.

10. Kitagaki $\mathrm{H}$, Takagi $\mathrm{H}$. Mitochondrial metabolism and stress response of yeast: applications in fermentation technologies. J Biosci Bioeng. 2014;117(4):383-93.

11. Bender T, Pena G, Martinou JC. Regulation of mitochondrial pyruvate uptake by alternative pyruvate carrier complexes. EMBO J. 2015;34(7):911-24.

12. Park SH, Kim S, Hahn JS. Improvement of isobutanol production in Saccharomyces cerevisiae by increasing mitochondrial import of pyruvate through mitochondrial pyruvate carrier. Appl Microbiol Biotechnol. 2016;100(17):7591-8.

13. Okamoto K, Kondo-Okamoto N, Ohsumi Y. Mitochondria-anchored receptor Atg32 mediates degradation of mitochondria via selective autophagy. Dev Cell. 2009;17(1):87-97.

14. Kanki T, Wang K, Cao Y, Baba M, Klionsky DJ. Atg32 is a mitochondrial protein that confers selectivity during mitophagy. Dev Cell. 2009;17(1):98-109.

15. Shiroma S, Jayakody LN, Horie K, Okamoto K, Kitagaki H. Enhancement of ethanol fermentation in Saccharomyces cerevisiae sake yeast by disrupting mitophagy function. Appl Environ Microbiol. 2014;80(3):1002-12.

16. Kim S, Hahn JS. Efficient production of 2,3-butanediol in Saccharomyces cerevisiae by eliminating ethanol and glycerol production and redox rebalancing. Metab Eng. 2015;31:94-101.

17. Lian JZ, Chao R, Zhao HM. Metabolic engineering of a Saccharomyces cerevisiae strain capable of simultaneously utilizing glucose and galactose to produce enantiopure (2R,3R)-butanediol. Metab Eng. 2014;23:92-9. 
18. Bialkowska AM. Strategies for efficient and economical 2,3-butanediol production: new trends in this field. World J Microbiol Biotechnol. 2016:32(12):200.

19. Xu Y, Chu H, Gao C, Tao F, Zhou Z, Li K, Li L, Ma C, Xu P. Systematic metabolic engineering of Escherichia coli for high-yield production of fuel bio-chemical 2,3-butanediol. Metab Eng. 2014;23:22-33.

20. Jurica MS, Mesecar A, Heath PJ, Shi W, Nowak T, Stoddard BL. The allosteric regulation of pyruvate kinase by fructose-1,6-bisphosphate. Structure. 1998:6:195-210.

21. Herzig S, Raemy E, Montessuit S, Veuthey JL, Zamboni N, Westermann B, Kunji ER, Martinou JC. Identification and functional expression of the mitochondrial pyruvate carrier. Science. 2012;337(6090):93-6.

22. Bricker DK, Taylor EB, Schell JC, OrsakT, Boutron A, Chen YC, Cox JE, Cardon CM, Van Vranken JG, Dephoure N, Redin C, Boudina S, Gygi SP, Brivet M, Thummel CS, Rutter J. A mitochondrial pyruvate carrier required for pyruvate uptake in yeast, Drosophila, and humans. Science. 2012;337(6090):96-100.

23. Vemuri GN, Eiteman MA, McEwen JE, Olsson L, Nielsen J. Increasing $\mathrm{NADH}$ oxidation reduces overflow metabolism in Saccharomyces cerevisiae. Proc Natl Acad Sci USA. 2007;104(7):2402-7.

24. Morita K, Nomura Y, Ishii J, Matsuda F, Kondo A, Shimizu H. Heterologous expression of bacterial phosphoenol pyruvate carboxylase and EntnerDoudoroff pathway in Saccharomyces cerevisiae for improvement of isobutanol production. J Biosci Bioeng. 2017;124(3):263-70.

25. Kajihata S, Matsuda F, Yoshimi M, Hayakawa K, Furusawa C, Kanda A, Shimizu H. ${ }^{13} \mathrm{C}$-based metabolic flux analysis of Saccharomyces cerevisiae with a reduced Crabtree effect. J Biosci Bioeng. 2015;120(2):140-4.

26. Kajihata S, Furusawa C, Matsuda F, Shimizu H. OpenMebius: an open source software for isotopically nonstationary ${ }^{13} \mathrm{C}$-based metabolic flux analysis. Biomed Res Int. 2014;2014:627014.
27. Antoniewicz MR, Kelleher JK, Stephanopoulos G. Determination of confidence intervals of metabolic fluxes estimated from stable isotope measurements. Metab Eng. 2006;8(4):324-37.

28. Kleijn RJ, van Winden WA, Ras C, van Gulik WM, Schipper D, Heijnen JJ. ${ }^{13} \mathrm{C}$-labeled gluconate tracing as a direct and accurate method for determining the pentose phosphate pathway split ratio in Penicillium chrysogenum. Appl Environ Microbiol. 2006;72(7):4743-54.

29. Okahashi N, Kajihata S, Furusawa C, Shimizu H. Reliable metabolic flux estimation in Escherichia coli central carbon metabolism using intracellular free amino acids. Metabolites. 2014;4(2):408-20.

30. Bolten JC, Kiefer P, Letisse F, Portais JC, Wittmann C. Sampling for metabolome analysis of microorganisms. Anal Chem. 2007;79:3843-9.

31. Kawase N, Tsugawa H, Bamba T, Fukusaki E. Different-batch metabolome analysis of Saccharomyces cerevisiae based on gas chromatography/mass spectrometry. J Biosci Bioeng. 2014;117:248-55.

32. Kato H, Izumi Y, Hasunuma T, Matsuda F, Kondo A. Widely targeted metabolic profiling analysis of yeast central metabolites. J Biosci Bioeng. 2012;113:665-73.

33. Ishii J, Izawa K, Matsumura S, Wakamura K, Tanino T, Tanaka T, Ogino C, Fukuda $\mathrm{H}$, Kondo A. A simple and immediate method for simultaneously evaluating expression level and plasmid maintenance in yeast. J Biochem. 2009;145:701-8.

34. Ishii J, Kondo T, Makino H, Ogura A, Matsuda F, Kondo A. Three gene expression vector sets for concurrently expressing multiple genes in Saccharomyces cerevisiae. FEMS Yeast Res. 2014;14:399-411.

\section{Publisher's Note}

Springer Nature remains neutral with regard to jurisdictional claims in published maps and institutional affiliations.
Ready to submit your research? Choose BMC and benefit from:

- fast, convenient online submission

- thorough peer review by experienced researchers in your field

- rapid publication on acceptance

- support for research data, including large and complex data types

- gold Open Access which fosters wider collaboration and increased citations

- maximum visibility for your research: over $100 \mathrm{M}$ website views per year

At BMC, research is always in progress.

Learn more biomedcentral.com/submissions 